\title{
铬铈相互作用对 $\mathrm{CrO}_{x}$-CeO $/$ Ti-PILC 催化剂上 NVOCs 催化降解性能的影响
}

\author{
杨姗姗, 黄琴琴, 周仁贤* \\ 浙江大学催化研究所, 杭州 310028 \\ *联系人, E-mail: zhourenxian@zju.edu.cn \\ 2014-03-13 收稿, 2014-04-21 接受, 2014-07-02 网络版发表 \\ 国家自然科学基金(21177110)资助
}

\begin{abstract}
摘要 采用等体积浸渍法制备了不同铬铈比例的 $\mathrm{CrO}_{x}-\mathrm{CeO}_{2} / \mathrm{Ti}-\mathrm{PILC}$ 催化剂 $(\mathrm{Cr} / \mathrm{Ce}$ 摩尔比 分别为 $1: 0,6: 1,4: 1,3: 1,0: 1$, 总负载量 $\mathrm{Cr}+\mathrm{Ce}$ 为 $8 \%$ (质量比)), 并研究了各催化剂上正 丁胺的催化降解性能. 结果表明, 随着 $\mathrm{Cr} / \mathrm{Ce}$ 比例的升高, 正丁胺的催化降解活性提高. 采用 $X$ 射线衍射 $(X R D) 、 X$ 射线光电子能谱 $(X P S)$ 、透射电子显微镜(TEM)、 $\mathrm{H}_{2}$ 程序升温 还原 $\left(\mathrm{H}_{2}-\mathrm{TPR}\right) 、 \mathrm{NH}_{3}$ 程序升温脱附 $\left(\mathrm{NH}_{3}-\mathrm{TPD}\right)$ 对不同铬铈比例催化剂的结构、氧化还原性 能、表面酸性质等进行了分析. 结果表明, 铬铈相互作用提高了催化剂上的酸性中心强度 和活性氧物种的流动性; 当催化剂中含有少量 $\mathrm{CeO}_{2}$ 时, 铈对铬的助催化作用更明显, 且 两者的协同作用加强，更有利于正丁胺的吸附和氧化.
\end{abstract}

\section{关键词}

催化氧化

$\mathrm{CrO}_{x}-\mathrm{CeO}_{2} / \mathrm{Ti}-\mathrm{PILC}$

正丁胺

铬铈比例
挥发性有机化合物(VOCs) 是一类常见的大气污 染物, 这类污染物对环境和人体健康有较大的危害. 随着我国工业化的迅速发展，工业排放 VOCs 由于其 量大、成分复杂等诸多因素, 特别是低浓度多组分 VOCs 的排放, 已经成为影响我国空气质量的主要污 染源之一 ${ }^{[1]}$. 其中含氮 $\operatorname{VOCs}(\mathrm{NVOCs})$ 是非常重要的 一类大气污染物, 可以分为胺类化合物、酰胺类化合 物、硝基类化合物和腈类化合物等. 这些化合物通常 以蒸汽的形式被人体吸人, 或者通过皮肤接触进人 人体, 对人体具有较大的毒害性. 因此研究和开发低 成本、高活性、高选择性和稳定性的 VOCs 氧化降解 催化剂是目前环境催化材料研究领域中极重要的课 题之一, 受到人们广泛的关注.

NVOCs 催化氧化的关键之处在于能够控制 $\mathrm{NO}_{x}$ 的生成量, 避免二次污染的产生. 催化燃烧技术具有 低能耗、高效率, 并且能有效避免二次污染等优
点 $^{[2 \sim 5]}$. 含氮有机废气催化燃烧的催化剂可以分为两 类，非贵金属氧化物催化剂和贵金属催化剂. 其中非 贵金属氧化物催化剂是以天然丝光沸石或 $\gamma-\mathrm{Al}_{2} \mathrm{O}_{3}$ 等 为载体, 负载 $\mathrm{Cr}, \mathrm{Cu}, \mathrm{Mn}, \mathrm{Co}$ 和 $\mathrm{Ni}$ 等活性组分的催化 剂，对各种 NVOCs 的完全转化温度一般在 280 $400^{\circ} \mathrm{C}$. 该类催化剂具有良好的控 $\mathrm{NO}_{x}$ 生成能力, 催 化剂上各种 NVOCs 的降解过程中都只产生少量的 $\mathrm{NO}_{x}^{[6 \sim 8]}$. 贵金属催化剂主要是以天然丝光沸石为载 体, 以贵金属 $\mathrm{Pt}$ 和 $\mathrm{Pd}$ 等作为活性组分的催化剂, 对 各种 NVOCs 的完全转化温度一般在 $250 \sim 300^{\circ} \mathrm{C}$. 罗 孟飞等人 ${ }^{[9]}$ 研究发现, 正丁胺在负载 $\mathrm{Pt}$ 的催化剂上 催化氧化时, $\mathrm{NO}_{x}$ 的浓度随反应温度升高会出现一个 最高点. 然而由于 $\mathrm{NO}_{x}$ 与还原性的中间产物正丁醇、 正丁醛等发生反应，并且 $\mathrm{NO}_{x}$ 自身会发生分解，因此 随着反应温度的进一步升高, $\mathrm{NO}_{x}$ 的浓度迅速下降. 与非贵金属氧化物催化剂相比，贵金属催化剂的控 catalysts for deep oxidation of $n$-butylamine. Chin Sci Bull, 2014, 59: 3987-3992, doi: 10.1007/s11434-014-0531-z 
$\mathrm{NO}_{x}$ 生成能力相对较差, 且 $\mathrm{NO}_{x}$ 的生成情况因反应物 种类的不同会呈现不同的规律. 传统含氮有机废气 氧化催化剂大都以天然丝光沸石或 $\gamma-\mathrm{Al}_{2} \mathrm{O}_{3}$ 等为载体, 本课题组尝试探索以柱撑黏土(PILCs)为载体合成铈 铬复合氧化物催化剂. PILCs 合成方法简单、成本低, 并且通过采用不同的交联剂和天然黏土, 可以调节 出具有不同物化性能的 PILCs, 以适应不同化学反应 中所需的活性和选择性 ${ }^{[9 \sim 11]}$. 常用的 PILCs 有铝、锆、 钛、铁、铬、铜混合物种合成柱撑黏土 ${ }^{[12,13]}$, 其中 Al-PILCs 和 Ti-PILCs 具有丰富的孔道结构和表面酸 性中心, 能为催化剂提供大的比表面积, 有利于活性 组分的分散, 促进反应物分子的吸附和活化, 且其孔 道结构对反应物和产物具有一定的择型作用, 从而 提高催化剂的活性及对反应终产物的选择性 ${ }^{[14,15]}$.

$\mathrm{CeO}_{2}$ 及铈基复合氧化物具有良好的储氧性能和 氧流动性, 在催化氧化-还原型反应中是不可或缺的 助催化剂成分, 它可提高催化剂的活性、选择性和稳 定性 ${ }^{[16 ~ 18]}$. 本文主要考察 $\mathrm{Cr}$ - $\mathrm{Ce}$ 之间的相互作用对 正丁胺催化降解性能的影响, 并采用 $\mathrm{X}$ 射线衍射 (XRD)、 $X$ 射线光电子能谱 $(X P S)$ 、透射电子显微镜 (TEM)、 $\mathrm{H}_{2}$ 程序升温还原 $\left(\mathrm{H}_{2}-\mathrm{TPR}\right) 、 \mathrm{NH}_{3}$ 程序升温脱 附 $\left(\mathrm{NH}_{3}-\mathrm{TPD}\right)$ 对不同铬铈比例催化剂的结构、氧化还 原性能、表面酸性质等进行了分析.

\section{1 实验}

(i ) 试剂与仪器. 硝酸亚铈 $\left(\mathrm{Ce}\left(\mathrm{NO}_{3}\right)_{3} \cdot 6 \mathrm{H}_{2} \mathrm{O}\right.$, $\mathrm{AR}$, 上海跃泾化工有限公司)、硝酸铬 $\left(\mathrm{Cr}\left(\mathrm{NO}_{3}\right)_{3}\right.$. $9 \mathrm{H}_{2} \mathrm{O}, \mathrm{CP}$, 国药集团化学试剂有限公司)、钛酸丁酯 $\left(\mathrm{Ti}\left(\mathrm{OC}_{4} \mathrm{H}_{9}\right)_{4}, \mathrm{CP}\right.$, 上海美兴化工有限公司 $) 、$ 氯化钠 $(\mathrm{NaCl}, \mathrm{AR}$, 宁波市化学试剂有限公司)、无水乙醇 $\left(\mathrm{C}_{2} \mathrm{H}_{5} \mathrm{OH}, \mathrm{AR}\right.$, 国药集团化学试剂有限公司)、氢氧化 钠 $(\mathrm{NaOH}, \mathrm{AR}$, 杭州萧山化学试剂厂) 、正丁胺 $\left(\mathrm{C}_{4} \mathrm{H}_{9} \mathrm{NH}_{2}, \mathrm{CP}\right.$, 上海业源联合化工有限公司 $)$. 催化 剂评价装置(WFS-3010, 天津先权仪器有限公司)、气 相色谱(GC-1690, 杭州科晓化工仪器设备有限公司).

(ii) 催化剂制备. 钠型蒙脱土 $(\mathrm{Na}-\mathrm{mmt})^{[11]}$ 的 制备: 以内蒙 $\mathrm{Ca}$ 型蒙脱土 $(>100$ 目, Ca-mmt) 为初始 材料, 该材料的分子式为 $\mathrm{Na}_{0.02} \mathrm{~K}_{0.02} \mathrm{Ca}_{0.39}\left[\mathrm{Fe}_{0.45} \mathrm{Mg}_{1.10}\right.$ $\left.\mathrm{Al}_{2.51}\right]\left[\mathrm{Si}_{7.91} \mathrm{Al}_{0.09}\right] \mathrm{O}_{20}(\mathrm{OH})_{4} \cdot n \mathrm{H}_{2} \mathrm{O}$, 其阳离子交换容 量 $(\mathrm{CEC})$ 为 $108.4 \mathrm{mg} / 100 \mathrm{~g}$ 土. $1.0 \mathrm{~mol} / \mathrm{L}$ 的 $\mathrm{NaCl}$ 溶液 与 $\mathrm{Ca}-\mathrm{mmt}(1 \mathrm{~g}$ 蒙脱土的 $\mathrm{NaCl}$ 溶液用量为 $100 \mathrm{~mL})$, $60^{\circ} \mathrm{C}$ 下搅拌 $3 \mathrm{~h}$ 后离心分离, $110^{\circ} \mathrm{C}$ 干燥.
Ti-PILC 的制备: 以 $\mathrm{Ti}\left(\mathrm{OC}_{4} \mathrm{H}_{9}\right)_{4}$ 为前驱体, 取 $34.5 \mathrm{~mL}$ 的 $\mathrm{Ti}\left(\mathrm{OC}_{4} \mathrm{H}_{9}\right)_{4}$ 加人到 $50 \mathrm{~mL}$ 无水乙醇中搅拌, 将上述溶液滴加到 $1 \mathrm{~mol} / \mathrm{L}$ 的 $\mathrm{HNO}_{3}$ 溶液中. 摚拌 1.5 $\mathrm{h}$, 用 $1 \mathrm{~mol} / \mathrm{L}$ 的 $\mathrm{NaOH}$ 调节 $\mathrm{pH} \approx 2$, 继续搅拌 $0.5 \mathrm{~h}$. 然后, 室温陈化 $12 \mathrm{~h}$ 得 $\mathrm{Ti}$ 交联剂. 以 $10 \mathrm{mmol} \mathrm{Ti} / \mathrm{g}$ clay 的比例将 $\mathrm{Ti}$ 交联剂滴加到质量分数为 $0.5 \%$ 的 $\mathrm{Na}-\mathrm{mmt}$ 溶液中, $20^{\circ} \mathrm{C}$ 下搅拌 $3 \mathrm{~h}$ 后离心水洗至中性, $110^{\circ} \mathrm{C}$ 干燥, $500^{\circ} \mathrm{C}$ 焙烧 $2 \mathrm{~h}$. 压片、粉碎、过篮后取 40 60 目的颗粒备用, 标记为 Ti-PILC(12,20).

$\mathrm{CrO}_{x}-\mathrm{CeO}_{2} / \mathrm{Ti}-\mathrm{PILC}$ 催化剂利用等体积浸渍法制 备. 以 Ti-PILC $(12,20)$ 为载体, 等体积浸渍定量的 $\mathrm{Cr}\left(\mathrm{NO}_{3}\right)_{3}$ 与 $\mathrm{Ce}\left(\mathrm{NO}_{3}\right)_{3}$ 的混合溶液, $110^{\circ} \mathrm{C}$ 干燥, $500^{\circ} \mathrm{C}$ 焙烧 $2 \mathrm{~h} . \mathrm{Cr}$ 和 $\mathrm{Ce}$ 的总负载量为 $8 \%$ (质量比), $\mathrm{Cr} / \mathrm{Ce}$ 的摩尔比例分别为 $1: 0,6: 1,4: 1,3: 1$ 和 $0: 1$, 催化剂 分别记为 $8 \mathrm{CrCe}(1: 0) / \mathrm{Ti}-\mathrm{PILC}(12,20), 8 \mathrm{CrCe}(6: 1) / \mathrm{Ti}-$ $\operatorname{PILC}(12,20), 8 \mathrm{CrCe}(4: 1) / \mathrm{Ti}-\mathrm{PILC}(12,20), 8 \mathrm{CrCe}(3: 1) /$ Ti-PILC(12,20)和 $8 \mathrm{CrCe}(0: 1) / \mathrm{Ti}-\operatorname{PILC}(12,20)$.

(iii) 表征. ARL X'TRA 型 XRD, $\mathrm{Cu} \mathrm{K} \alpha$ 射线, 管电压 $40 \mathrm{kV}$, 管电流 $40 \mathrm{~mA}$, 扫描速度 $0.02 \%$, 黏 土材料负载型催化剂的扫描范围为 $20^{\circ} \sim 80^{\circ}$. 催化剂 的表面元素含量及结合能在 PHI 5000c 型 XPS/UPS 上测定, $\mathrm{Al} \mathrm{K \alpha}$ 射线, 电压 $12.5 \mathrm{kV}$, 电子能量 1486.6 $\mathrm{eV}$, 所有的谱图用 $\mathrm{C} 1 \mathrm{~s}$ 的结合能 $(284.6 \mathrm{eV})$ 作为基准 进行校正.

JEM-2010 型 TEM, 工作电压 $200 \mathrm{Kv}$, 将样品粉 末分散于无水乙醇中, 超声振荡 $5 \mathrm{~min}$, 用镀有碳膜 的铜网捞取悬浮样品, 待干燥后装人电子显微镜预 处理室, 抽空后转人测量室, 观察形貌并摄取照片.

$\mathrm{H}_{2}$-TPR 测定: 称取 $100 \mathrm{mg}$ 催化剂置于石英反应 管中, 空气气氛中 $300^{\circ} \mathrm{C}$ 预处理 $0.5 \mathrm{~h}$, 冷却至 $30^{\circ} \mathrm{C}$. 然后将气氛切换至 $5 \% \mathrm{H}_{2} / \mathrm{Ar}$ (体积比)的混合气 (40 $\mathrm{mL} / \mathrm{min}$ ), 至基线稳定后以 $10^{\circ} \mathrm{C} / \mathrm{min}$ 的升温速率进行 程序升温. $\mathrm{H}_{2} / \mathrm{Ar}$ 混合气预先用 $5 \mathrm{~A}$ 分子篮和 HDY 型 脱氧催化剂分别进行脱水和脱氧净化.

$\mathrm{NH}_{3}$-TPD 测定: 称取 $100 \mathrm{mg}$ 样品置于石英反应 管中, 高纯 $\mathrm{N}_{2}$ (纯度 $99.99 \%$ )气氛中 $500^{\circ} \mathrm{C}$ 下预处理 $0.5 \mathrm{~h}$, 冷却至 $100^{\circ} \mathrm{C}$. 将气氛切换至 $20 \% \mathrm{NH}_{3} / \mathrm{N}_{2}$ (体积 比)的混合气 $(30 \mathrm{~mL} / \mathrm{min})$ 处理 $0.5 \mathrm{~h}$, 再将气氛切换至 高纯 $\mathrm{N}_{2}(40 \mathrm{~mL} / \mathrm{min})$ 直至物理吸附的 $\mathrm{NH}_{3}$ 吹扫完成后, 以 $10^{\circ} \mathrm{C} / \mathrm{min}$ 的升温速率从 $100^{\circ} \mathrm{C}$ 开始程序升温检测.

(iv) 催化剂活性评价方法. 催化剂活性评价在 WFS-3010 上进行. 量取 40 60 目的催化剂 $0.3 \mathrm{~mL}$, 
控制反应空速为 $20000 \mathrm{~h}^{-1}$, 反应物浓度为 $1000 \mathrm{ppm}$ $(1 \mathrm{ppm}=1 \mathrm{mg} / \mathrm{L})$ 左右. 反应物和产物通过六通阀定量 $(0.5 \mathrm{~mL})$ 进样, 反应器进出口中 NVOCs 的浓度用 GC-1690 气相色谱仪 (FID) 在线检测 (汽化室温度 $180^{\circ} \mathrm{C}$, 柱温 $120^{\circ} \mathrm{C}$, 检测器温度 $200^{\circ} \mathrm{C}$ ), 由 $\mathrm{N} 2000$ 色 谱工作站记录分析数据.

\section{2 结果与讨论}

\section{1 活性评价}

图 1 所示为不同铬铈比例催化剂上正丁胺催化 降解的转化率曲线. 由图 1 可以看出, 各催化剂对正 丁胺的催化降解能力为: $8 \mathrm{CrCe}(6: 1) / \mathrm{Ti}-\mathrm{PILC}(12,20)>$ $8 \mathrm{CrCe}(4: 1) / \mathrm{Ti}-\mathrm{PILC}(12,20)>8 \mathrm{CrCe}(3: 1) / \mathrm{Ti}-\mathrm{PILC}(12$, $20)>8 \mathrm{CrCe}(1: 0) / \mathrm{Ti}-\mathrm{PILC}(12,20)>>8 \mathrm{CrCe}(0: 1) / \mathrm{Ti}-$ $\operatorname{PILC}(12,20)$. 与负载单活性组分的催化剂相比, 在 负载 $\mathrm{CrO}_{x}-\mathrm{CeO}_{2}$ 双组分催化剂上正丁胺的催化降解 活性明显提高, 这说明 $\mathrm{Cr}-\mathrm{Ce}$ 相互作用有利于催化剂 对正丁胺的降解. 此外, 随着 $\mathrm{Cr} / \mathrm{Ce}$ 比例的升高, 催 化剂对正丁胺的催化降解活性升高, 说明当活性组 分中铈的含量较低时, 铈对铬的助催化作用更明显, 两者之间的相互作用有助于提高催化剂的活性.

NVOCs 催化氧化的关键之处在于能够控制 $\mathrm{NO}_{x}$ 的生成量, 避免二次污染的产生, 图 2 所示为不同铬 铈比例催化剂上正丁胺催化降解时 $\mathrm{NO}_{x}$ 的生成情况. 由图 2 可以看出当正丁胺的转化率达到 $98 \%$ 时, 各催 化剂上 $\mathrm{NO}_{x}$ 的产率均小于 $0.5 \%$, 并且当反应温度再 升高 $50^{\circ} \mathrm{C}$ 时, 各催化剂上 $\mathrm{NO}_{x}$ 的产率依然低于 $1 \%$,

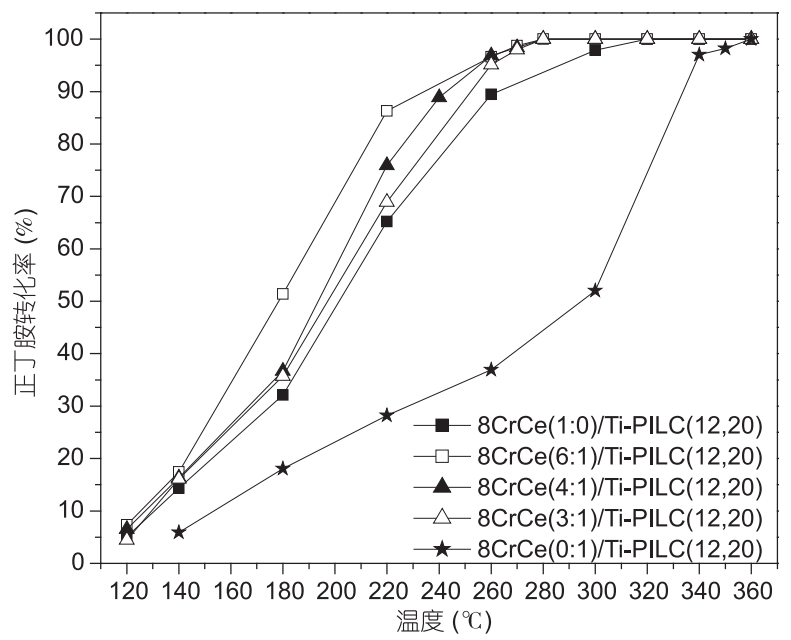

图 $18 \mathrm{CrCe} / \mathrm{Ti}$-PILC $(12,20)$ 催化剂上正丁胺催化降解的 转化率曲线

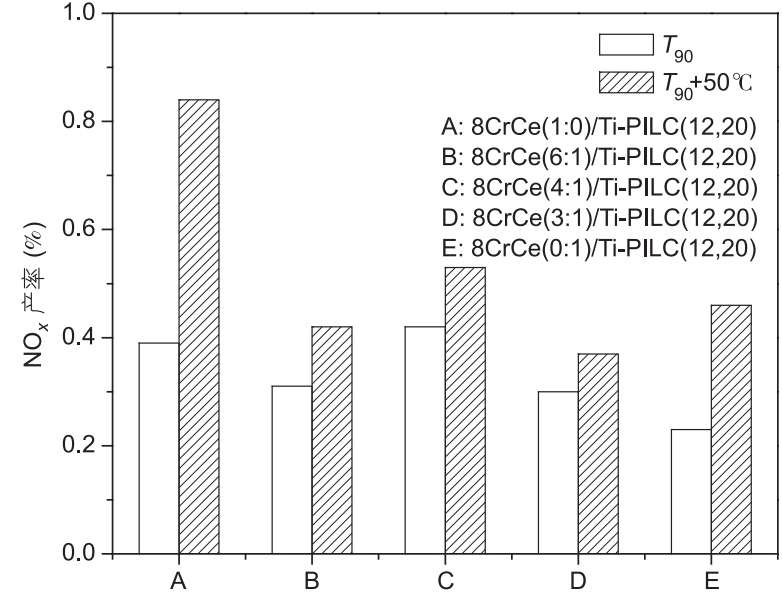

图 $28 \mathrm{CrCe}$ /Ti-PILC $(12,20)$ 催化剂上正丁胺催化降解的

$$
\mathrm{NO}_{x} \text { 产率 }
$$

说明所有的催化剂都表现出良好的控 $\mathrm{NO}_{x}$ 生成能力.

\section{2 织构-结构分析}

不同铬铈比例催化剂的 XRD 谱图如图 3 所示. 由图 3 可看出, 负载单组分铈的催化剂上出现了明显 的 $\mathrm{CeO}_{2}$ 特征衍射峰, 活性相 $\mathrm{CeO}_{2}$ 在载体表面发生了 严重的颗粒团聚. 而在负载铬铈双组分的催化剂上 没有明显的 $\mathrm{CeO}_{2}$ 特征峰出现, 说明 $\mathrm{Cr}-\mathrm{Ce}$ 相互作用 抑制了 $\mathrm{CeO}_{2}$ 在载体表面颗粒团聚的发生. 所有含铬 的催化剂上都出现了明显的 $\mathrm{Cr}_{2} \mathrm{O}_{3}$ 特征衍射峰 ${ }^{[19]}$, 且 随着 $\mathrm{Cr} / \mathrm{Ce}$ 比例的升高, $\mathrm{Cr}_{2} \mathrm{O}_{3}$ 特征峰的强度逐渐增 强, 但当 $\mathrm{Cr} / \mathrm{Ce}$ 比例从 $6: 1$ 上升到 $1: 0$ 时, 催化剂上 $\mathrm{Cr}_{2} \mathrm{O}_{3}$ 特征峰的强度基本保持不变.

由 TEM 图片(图 4)可知, $8 \mathrm{CrCe}(1: 0) / \mathrm{Ti}-\mathrm{PILC}(12$,

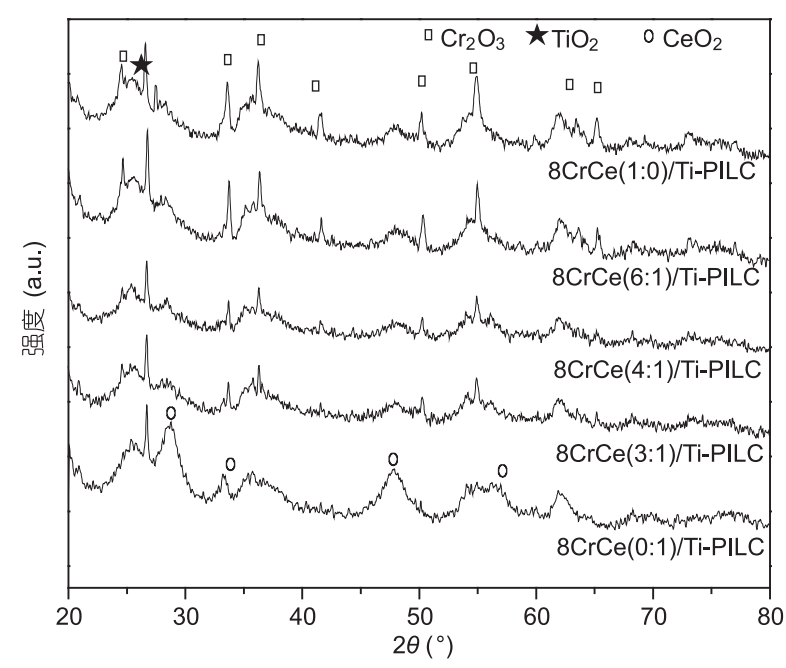

图 $38 \mathrm{CrCe}$ /Ti-PILC $(12,20)$ 催化剂的 XRD 谱图 


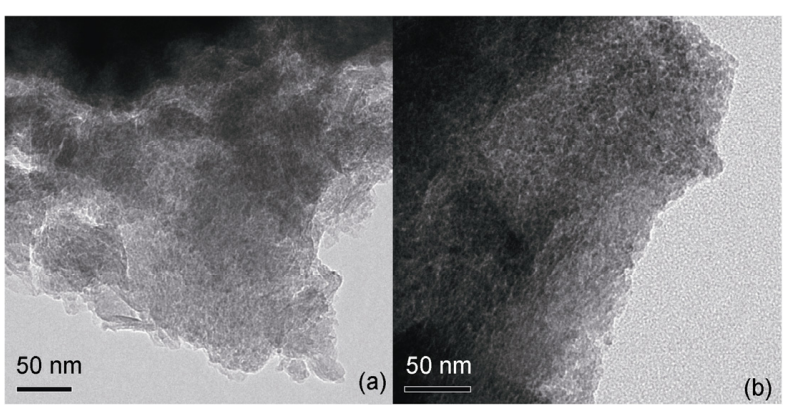

图 $48 \mathrm{CrCe} / \mathrm{Ti}-\mathrm{PILC}(12,20)$ 催化剂的 TEM 图

(a) $8 \mathrm{CrCe}(1: 0) / \mathrm{Ti}-\mathrm{PILC}(12,20)$; (b) $8 \mathrm{CrCe}(6: 1) / \mathrm{Ti}-\mathrm{PILC}(12,20)$

20)和 $8 \mathrm{CrCe}(6: 1) / \mathrm{Ti}-\mathrm{PILC}(12,20)$ 上都出现了大量而 密集的活性组分颗粒，而且它们在载体上的分散效 果差别不大. 结合活性评价结果，我们认为活性相在 催化剂表面的分散效果与催化剂的活性之间没有必 然的联系，负载铬铈双组分催化剂的活性明显高于 单活性组分催化剂，这可能与铬铈之间的相互作用 强弱有关.

图 5 所示为不同铬铈比例催化剂的 XPS 谱图, 表 1 列出了催化剂上主要元素的结合能和相对含量 数值. 由图 5(a)和表 1 可知, 所有的催化剂上都出现 了 $\mathrm{Cr}^{3+}$ 和 $\mathrm{Cr}^{6+}$ 的特征谱线 ${ }^{[20,21]} \cdot \mathrm{Cr}^{6+}$ 物种具有更强的
氧化性, 更有利于反应物的氧化. 与单铬催化剂相比, 负载铬铈双组分的催化剂上 $\mathrm{Cr} 2 \mathrm{p}_{3 / 2}$ 的结合能增加, 说明 $\mathrm{Cr}-\mathrm{Ce}$ 相互作用提高了 $\mathrm{Cr}-\mathrm{O}$ 键的键合能力. 但 $\mathrm{Cr}^{3+} / \mathrm{Cr}^{6+}$ 的比值升高, 这可能是由于随着催化剂中 $\mathrm{Cr}$ 负载量的降低 $\mathrm{Cr}^{6+}$ 较难形成. 从 $\mathrm{O} 1 \mathrm{~s}$ 的结合能数据 可知, 各催化剂上 $\mathrm{O} 1 \mathrm{~s}$ 的结合能都在 $531.6 \sim 532.0 \mathrm{eV}$, 说明这些氧是与氧空穴或者 $\mathrm{OH}$ 基团有关的氧物种, 此类氧物种具有良好的迁移能力, 对氧化降解反应 的活性较高 ${ }^{[2227]}$. 由图 5(b)和表 1 可知, 随着 $\mathrm{Cr} / \mathrm{Ce}$ 比例的升高, $\mathrm{Ce}^{3+} / \mathrm{Ce}^{4+}$ 的比值也升高, $\mathrm{Cr}-\mathrm{Ce}$ 相互作用 有利于催化剂中氧空穴的形成 ${ }^{[23,28]}$. 结合活性评价 结果, 可知 $8 \mathrm{CrCe}(6: 1) / \mathrm{Ti}-\mathrm{PILC}(12,20)$ 上氧空穴的含 量和 $\mathrm{Cr}^{6+}$ 的含量相对较高, 有利于氧的吸附及反应物 的深度氧化, 因此该催化剂对正丁胺的催化活性最高.

\section{3 氧化还原性能分析}

图 6 所示为不同铬铈比例催化剂的 $\mathrm{H}_{2}$-TPR 谱图. 由图可知, 负载单组分铈的催化剂只在 $500^{\circ} \mathrm{C}$ 以上出 现了 3 个较弱的还原峰, 分别为 $\mathrm{CeO}_{2}$ 表面氧的还原 峰( $\beta$ 和 $\gamma$ 峰) 以及 $\mathrm{CeO}_{2}$ 体相氧与黏土中 $\mathrm{Fe}$ 物种的还 原峰; 负载单组分铬的催化剂在 $300^{\circ} \mathrm{C}$ 出现了 1 个强

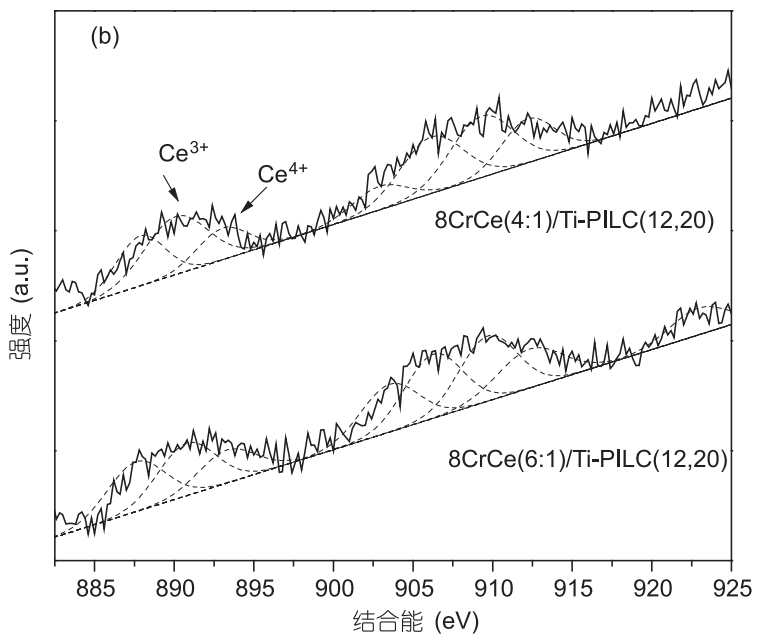

图 5 不同铬铈比例 $8 \mathrm{CrCe} / \mathrm{Ti}-\mathrm{PILC}(12,20)$ 催化剂的 XPS 谱图

(a) $\mathrm{Cr} 2 \mathrm{p}$; (b) $\mathrm{Ce} 3 \mathrm{~d}_{5 / 2}$

表 1 催化剂上主要元素的结合能和相对含量

\begin{tabular}{cccccc}
\hline \multirow{2}{*}{ 催化剂 } & \multicolumn{3}{c}{ 结合能 $(\mathrm{eV})$} & \multicolumn{2}{c}{ 相对含量(\%) } \\
\cline { 2 - 6 } & \multicolumn{3}{c}{$\mathrm{Cr} 2 \mathrm{p}_{3 / 2}$} & $\mathrm{O} \mathrm{1s}$ & \multicolumn{2}{c}{$\mathrm{Cr}^{3+} / \mathrm{Cr}^{6+}$} & $\mathrm{Ce}^{3+} / \mathrm{Ce}^{4+}$ \\
\hline $8 \mathrm{CrCe}(1: 0) /$ Ti-PILC(12,20) & 577.2 & 581.8 & 531.6 & 2.28 & - \\
$8 \mathrm{CrCe}(6: 1) /$ Ti-PILC(12,20) & 577.8 & 582.6 & 532.0 & 3.29 & 1.59 \\
$8 \mathrm{CrCe}(4: 1) /$ Ti-PILC(12,20) & 577.8 & 582.8 & 531.8 & 3.59 & 1.72 \\
\hline
\end{tabular}




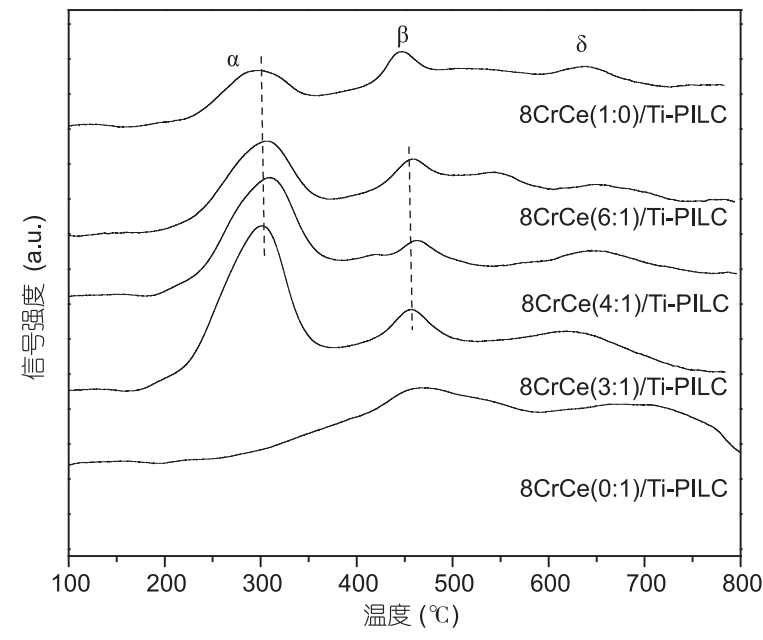

图 $68 \mathrm{CrCe} / \mathrm{Ti}-P I L C(12,20)$ 催化剂的 $\mathrm{H}_{2}$-TPR 谱图

还原峰 ( $\alpha$ 峰), 可归属为 $\mathrm{Cr}^{6+}$ 物种的还原峰 ${ }^{[29,30]}$. 根 据相关文献[31 34]报道, $\mathrm{CeO}_{2}$ 的还原峰高于 $350^{\circ} \mathrm{C}$, 其中铬的存在有利于 VOCs 的催化降解, 且其还原峰 温度低于 $300^{\circ} \mathrm{C}$. 与单铬催化剂相比, $8 \mathrm{CrCe}(6: 1) / \mathrm{Ti}-$ $\operatorname{PILC}(12,20)$ 具有最高的反应活性, 可能是因为 $\mathrm{Cr}^{6+}$ 的含量相对较高, 这与 XPS 的结果相对应. 随着 $\mathrm{Cr}$ 含量的增加, 催化剂上活性氧物种的流动性增强, 催 化剂的氧化还原性能提高. $8 \mathrm{CrCe}(6: 1) / \mathrm{Ti}-\mathrm{PILC}(12,20)$ 上既具有适量的主活性组分 $\mathrm{CrO}_{x}$, 又具有较好的氧 流动性, 因此其对正丁胺的催化降解活性最高.

\section{4 表面酸性质分析}

不同铬铈比例催化剂的 $\mathrm{NH}_{3}$-TPD 谱图如图 7 所 示. 各催化剂 $\mathrm{NH}_{3}$ 脱附峰的强度没有明显差别, 说明 $\mathrm{Cr}-\mathrm{Ce}$ 相互作用对催化剂上酸性中心数目的变化没有 明显影响. 但是, 随着 $\mathrm{Cr} / \mathrm{Ce}$ 比例的升高, 各脱附峰 的峰顶温度(表 2)逐渐往高温偏移, 说明 $\mathrm{Cr}-\mathrm{Ce}$ 相互 作用提高了催化剂上酸性中心的强度, 有利于对正 丁胺的催化降解活性, 这与活性评价结果相一致.

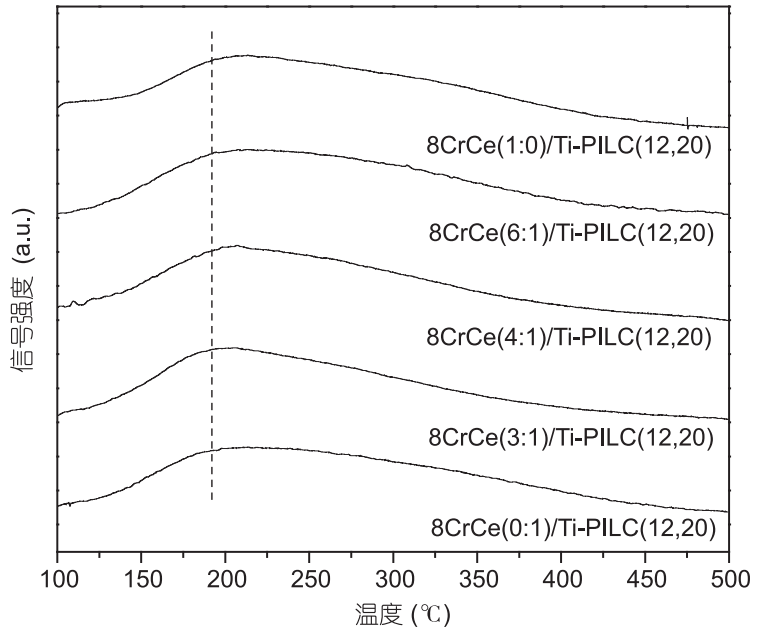

图 $78 \mathrm{CrCe} / \mathrm{Ti}-\mathrm{PILC}(12,20)$ 催化剂的 $\mathrm{NH}_{3}$-TPD 谱图

表 2 催化剂脱附峰温度

\begin{tabular}{cc}
\hline 催化剂 & 峰顶温度 $\left({ }^{\circ} \mathrm{C}\right)$ \\
\hline $8 \mathrm{CrCe}(1: 0) / \operatorname{Ti}-\operatorname{PILC}(12,20)$ & 210 \\
$8 \mathrm{CrCe}(6: 1) / \mathrm{Ti}-\operatorname{PILC}(12,20)$ & 212 \\
$8 \mathrm{CrCe}(4: 1) / \mathrm{Ti}-\operatorname{PILC}(12,20)$ & 207 \\
$8 \mathrm{CrCe}(3: 1) / \mathrm{Ti}-\operatorname{PILC}(12,20)$ & 200 \\
$8 \mathrm{CrCe}(0: 1) / \mathrm{Ti}-\operatorname{PILC}(12,20)$ & 196 \\
\hline
\end{tabular}

\section{3 结论}

$\mathrm{Cr}-\mathrm{Ce}$ 相互作用提高了催化剂上的酸性中心的强 度和活性氧物种的流动性, 有利于正丁胺的吸附和 氧化, 因此负载铬铈双组分催化剂对正丁胺的催化 降解活性明显高于其负载单组分的催化剂. 当催化 剂中含有适量 $\mathrm{CeO}_{2}$ 时, 助催化作用更明显, 并且能 显著提高铬铈之间的协同作用. 此外, 铬铈双组分催 化剂具有良好的控 $\mathrm{NO}_{x}$ 生成能力, 当正丁胺的转化 率达到 $98 \%$ 时, 各催化剂上 $\mathrm{NO}_{x}$ 的产率均小于 $0.5 \%$, 有效地避免了二次污染，具有很大的现实意义.

\section{参考文献}

1 López-Fonseca R, Gutiérrez-Ortiz J I, Gutierrez-Ortiz M A, et al. Dealuminated Y zeolites for destruction of chlorinated volatile organic compounds. J Catal, 2002, 209: 145-150

2 Dai J L, Zhang M, Hu Q H, et al. Adsorption and desorption of iodine by various Chinese soils: II. Iodide and iodate. Geoderma, 2009, 153: $130-135$

3 Bertinchamps F, Treinen M, Blangenois $\mathrm{N}$, et al. Positive effect of $\mathrm{NO}_{x}$ on the performances of $\mathrm{VO}_{x} / \mathrm{TiO}_{2}$-based catalysts in the total oxidation abatement of chlorobenzene. J Catal, 2005, 230: 493-498

4 Liu X W, Su Y Q, Luo L S, et al. Effect of hydrogen treatment on solidification structures and mechanical properties of TiAl alloys. Int J Hydrog Energy, 2011, 36: 3260-3267 
5 Greene H L, Brown B I, McClenathan D T, et al. A new variant of type IV glycogenosis: Deficiency of branching enzyme activity without apparent progressive liver disease. Hepatology, 1988, 8: 302-306

6 Dai Y, Wang X Y, Li D, et al. Catalytic combustion of chlorobenzene over Mn-Ce-La-O mixed oxide catalysts. J Hazard Mater, 2011, 188: 132-139

7 罗孟飞, 袁贤金陈敏. Ce-Cr 硅藻土对集中低分子量有机物氧化作用的催化活性研究. 环境科学, 1992, 14: 17-19

8 袁贤金金, 蒋欣凡, 王莉红. 含氮有机物深度氧化催化剂的研究. 石油化工, 1990, 19: 828-832

9 罗孟飞, 袁贤崟, 王逸民. 正丁胺在 Pt/NM 催化剂上完全氧化反应的研究. 化学反应工程与工艺, 1999, 9: 114-117

10 罗孟飞, 袁贤釒生. 正丁胺在 Pt/NM 催化剂上完全氧化反应机理的研究. 环境科学学报, 1994, 14: 355-360

11 Yuan P, Annabi-Bergaya F, Tao Q, et al. A combined study by XRD, FTIR, TG and HRTEM on the structure of delaminated Fe-intercalated/pillared clay. J Colloid Interface Sci, 2008, 324: 142-149

12 Brindley G W, Sempels R E. Preparation and properties of some hydroxy-aluminium beidellites. Clay Miner, 1977, 12: 229-237

13 Huang Q Q, Zuo S F, Zhou R X. Catalytic performance of pillared interlayered clays (PILCs) supported CrCe catalysts for deep oxidation of nitrogen-containing VOCs. Appl Catal B Environ, 2010, 95: 327-334

14 Liu G L, Mei H W, Yu X Q, et al. QTL analysis of panicle neck diameter, a trait highly correlated with panicle size, under well-watered and drought conditions in rice. Plant Sci, 2008, 174: 71-77

15 Frost R L, Ding Z, Kloprogge J T, et al. Thermal stability of azurite and malachite in relation to the formation of mediaeval glass and glazes. Thermochim Acta, 2002, 390: 133-144

16 Zimmer P, Tschöpe A, Birringer R. Temperature-programmed reaction spectroscopy of ceria-and Cu/ceria-supported oxide catalyst. J Catal, 2002, 205: 339-345

17 Kröll M, Blau W J, Grandjean D, et al. Magnetic properties of ferromagnetic nanowires embedded in nanoporous alumina membranes. J Magn Magn Mater, 2002, 249: 241-245

18 Shishido T, Sukenobu M, Morioka H, et al. Partial oxidation of methane over Ni/Mg-Al oxide catalysts prepared by solid phase crystallization method from Mg-Al hydrotalcite-like precursors. Appl Catal A Gen, 2002, 223: 35-42

19 Zhu P, Li J, Zuo S, et al. Preferential oxidation properties of $\mathrm{CO}$ in excess hydrogen over $\mathrm{CuO}-\mathrm{CeO}_{2}$ catalyst prepared by hydrothermal method. Appl Surf Sci, 2008, 255: 2903-2909

20 Oliveira L C A, Lago R M, Fabris J D, et al. Catalytic oxidation of aromatic VOCs with Cr or Pd-impregnated Al-pillared bentonite: Byproduct formation and deactivation studies. Appl Clay Sci, 2008, 39: 218-222

21 Słoczyński J, Janas J, Machej T, et al. Catalytic activity of chromium spinels in SCR of NO with $\mathrm{NH}_{3}$. Appl Catal B Environ, 2000, 24: 45-60

22 Lenarda M, Storaro L, Talon A, et al. Synthesis, characterization and catalytic activity of aluminum pillared synthetic Ti-, Fe-or Zn-substituted smectites. Stud Surf Sci Catal, 1998, 118: 959-966

23 Wu X D, Fan Y Z, Wu J S. A study on the variations of the electrical resistance for NiTi shape memory alloy wires during the thermomechanical loading. Mater Des, 2000, 21: 511-515

24 Ponce S, Pena M A, Fierro J L G. Surface properties and catalytic performance in methane combustion of Sr-substituted lanthanum manganites. Appl Catal B Environ, 2000, 24: 193-205

25 Zhang-Steenwinkel Y, Beckers J, Bliek A. Surface properties and catalytic performance in CO oxidation of cerium substituted lanthanummanganese oxides. Appl Catal A Gen, 2002, 235: 79-92

$26 \mathrm{Yu}$ Z, Gao L, Yuan S, et al. Solid defect structure and catalytic activity of perovskite-type catalysts $\mathrm{La}_{1-x} \mathrm{Sr}_{x} \mathrm{NiO}_{3-\lambda}$ and $\mathrm{La}_{1-1.333 x} \mathrm{Th}_{x} \mathrm{NiO}_{3-\lambda}$. J Chem Soc Faraday Trans, 1992, 88: 3245-3249

27 Chen H Y, Sayari A, Adnot A, et al. Composition-activity effects of Mn-Ce-O composites on phenol catalytic wet oxidation. Appl Catal B Environ, 2001, 32: 195-204

28 Praline G, Koel B E, Hance R L, et al. X-ray photoelectron study of the reaction of oxygen with cerium. J Electron Spectrosc Relat Phenom 1980, 21: 17-30

29 Concepción P, Corma A, López Nieto J M, et al. Selective oxidation of hydrocarbons on V-and/or Co-containing aluminophosphate (MeAPO-5) using molecular oxygen. Appl Catal A Gen, 1996, 143: 17-28

30 Chen Y C, Cheng K C, Choong W S, et al. A high-throughput data acquisition system for the HyperCP experiment. Nucl Instrum Methods Phys Res Sect A, 2000, 455: 424-432

31 Caputo T, Lisi L, Russo G. On the role of redox properties of $\mathrm{CuO} / \mathrm{CeO}_{2}$ catalysts in the preferential oxidation of $\mathrm{CO}$ in $\mathrm{H}_{2}$-rich gases. Appl Catal A Gen, 2008, 348: 42-53

32 Primet M, Garbowski E. Catalysis by ceria and related materials. In: Trovarelli A, ed. Catalysis science series, vol. 2. London: Imperial College Press, 2002

33 Pintar A, Batista J. Chromatic numbers and cycle parities of quadrangulations on nonorientable closed surfaces. J Colloid Interface Sci, 2005, 285: 218-231

34 Ratnasamy P, Srinivas D, Satyanarayana C V V, et al. Correlation of surface, mechanical and microproperties of tetrahedral amorphous carbon films deposited under different magnetic confinement conditions. Appl Surf Sci, 2004, 221: 455-465 